\title{
Correlation Brain Signals and Tractor Sound Signals Based on Fractal Analysis
}

\author{
Majid Lashgari ${ }^{1 *}$ (D), Mohammadreza Arab², Mohsen Nadjafi ${ }^{3}$, Ali Maleki ${ }^{4}$
}

1. Associate Professor, Department of Biosystems Engineering, Arak University, Arak, Iran

2. PhD, Department of Medical Engineering, Arak University of Medical Sciences, Arak, Iran

3. Assistant Professor, Department of Electrical Engineering, Arak University of Technology, Arak, Iran

4. Associate Professor, Department of Biosystems Engineering, Shahrekord University, Shahrekord, Iran

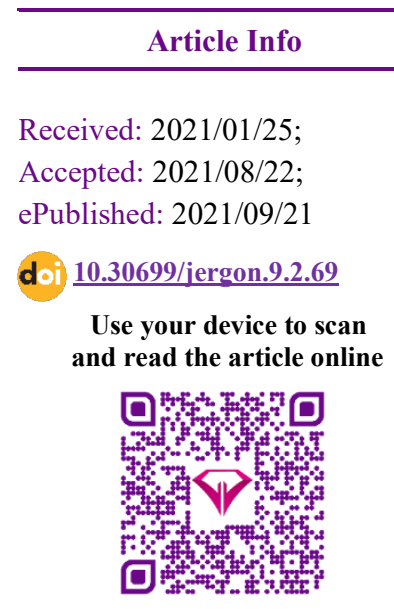

Corresponding Author Majid Lashgari Associate Professor, Department of Biosystems Engineering, Arak University, Arak, Iran Email: m-lashgari@araku.ac.ir

\section{ABSTRACT}

Background and Objectives: Due to the sound caused by various machines and tools in different agriculture sectors, occupational safety and health should be continuously evaluated. Indeed, the harmful effects of sound can be better reduced when the effects of sound on people's health and performance are fully known.

Methods: In this study, a garden tractor was used. Sixteen volunteers were exposed to the sound of the tractor, and their EEG was recorded at four different engine speeds. Then, Higuchi and Katz methods were used to calculate the fractal dimension of sound signals as well as brain signals.

Results: The results showed that by increasing engine speed, the values of the fractal dimension in both Higuchi and Katz methods increased. The results also showed an increase in the fractal dimension of brain signals due to an increase in engine speed. The regression results also showed a high correlation between the two brain signals and the sound. The coefficient of explanation was 0.896 and 0.859 in Higuchi and Katz methods, respectively.

Conclusion: This study showed that people's reactions, when exposed to sound, can be predicted using the fractal dimension. Therefore, it is possible to estimate the characteristics of brain signals without recording them, which are often costly and time-consuming.

Keywords: EEG, Higuchi, Katz, Tractor, Sound

How to Cite This Article:

Lashgari M, Arab M, Nadjafi M, Maleki A. Correlation Brain Signals and Tractor Sound Signals Based on Fractal Analysis. Iran J Ergon. 2021; 9(2):69-81 


\section{Extended Abstract}

\section{Introduction}

Due to the sound caused by various machines and tools in different agriculture sectors, occupational safety and health should be continuously evaluated. Among the most important adverse effects of sound on humans, in addition to permanent and temporary hearing loss, we can mention such things as reduced work efficiency and increased risk of accidents, sleep disorders, neurological and mental disorders and cardiovascular disease [4-9]. This phenomenon has also had detrimental effects on agricultural activists [10]. Indeed, the harmful effects of sound can be better reduced when the effects of sound on people's health and performance are fully known.

Given that a group of agricultural activists who deal with a variety of machinery are directly exposed to sound, it is absolutely necessary to be aware of the effects of sound on this occupational group. Although studies have been conducted on the relationship between the fractal dimension of external stimuli and the types of human biological signals, according to the research, no studies have been conducted on this topic in the country, especially in the agricultural sector, or to be more precise, no results have been published. Consid-

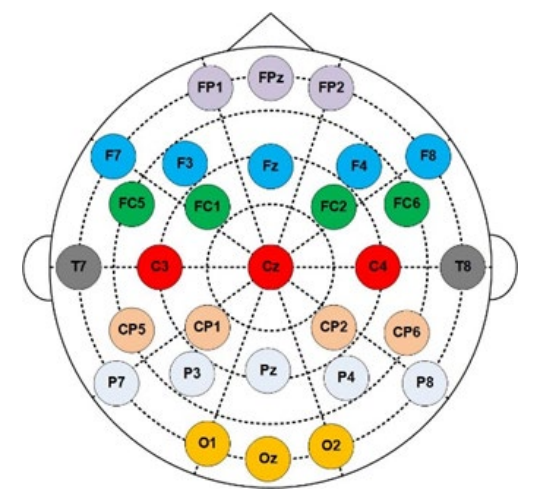

ering that tractors are used in most agricultural affairs and most of the common tractors in the country do not have cabins, the aim of this study was to investigate the relationship between sound as an external stimulus that most tractor drivers are exposed to and their reaction during work with Use brain activity assessment.

\section{Methods}

In this study, a garden tractor was used. Sixteen volunteers were exposed to the sound of the tractor, and their EEG was recorded at four different engine speeds. Then, Higuchi and Katz methods were used to calculate the fractal dimension of sound signals as well as brain signals.

\section{Results}

The results showed that by increasing engine speed, the values of the fractal dimension in both Higuchi and Katz methods increased. The results also showed an increase in the fractal dimension of brain signals due to an increase in engine speed. The regression results also showed a high correlation between the two brain signals and the sound. The coefficient of explanation was 0.896 and 0.859 in Higuchi and Katz methods, respectively.

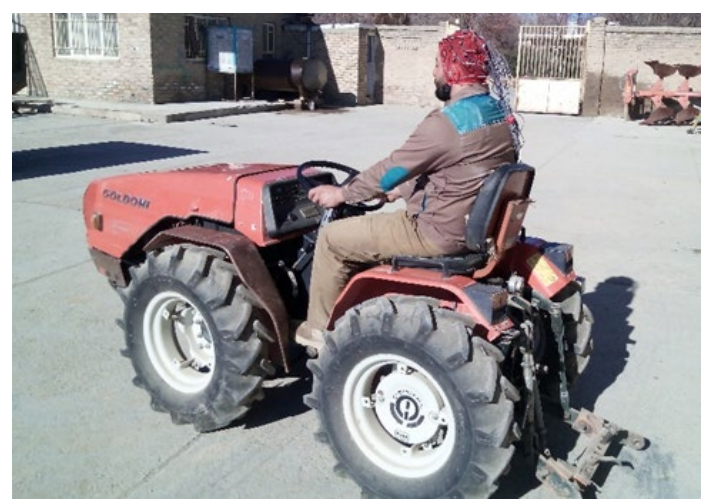

Figure 1. EEG recording using a standard cap 10-20 (right) and the position of the electrodes on the head (left)

\section{Discussion}

The results of this study indicate that the fractal dimension of tractor sound signals increases with increasing engine speed, and this increase can be seen in both Katz and Higuchi methods. In general, with increasing engine speed, the speed of move- ment of engine parts and components increases on the one hand, and on the other hand, the vibrational movements of other tractor components increase. For this reason, in various studies, an increase in sound pressure level has been reported in exchange 
for an increase in engine speed $[37,36]$. Given that increasing the sound pressure level is associated with more irregularity of the sound signal, increaseing the fractal dimension of the sound signal seems reasonable [26].

Given that the volunteers participating in this study were only exposed to motor noise, changes in the fractal dimension of brain signals can be attributed to changes in sound. The effect of sound on human brain activity has also been reported in other studies. Research has shown that human exposure to pleasant and unpleasant tones in different areas of the brain is effective [38]. Noisy work environment can also cause changes in brain signals [39]. As mentioned earlier, the purpose of this study is to investigate the relationship between voice and brain response in individuals. For this purpose, regression was performed between the fractal dimension of brain signals and sound. Although a strong and significant correlation was observed between HFD brain signals and sound, no such conclusion was reached for KFD. This indicates that the method of determining the fractal dimension is important that in other researches, different results of the methods of determining the fractal dimension have been mentioned $[41,40]$. However, the high accuracy of Higuchi method in estimating the fractal dimension compared to other methods such as Katz has been confirmed in other researches [32].

The existence of a correlation between the fractal dimension of external stimuli and the reaction of people who are exposed to those stimuli has also been mentioned in some studies $[42,30]$. As the results of this study show, with the increase in the fractal dimension of the Higuchi sound as an external stimulus, the fractal dimension of the brain signal also increased. In fact, increasing the complexity of sound has increased the complexity of brain signals, which is consistent with the results of other research [30]. After confirming the existence of a strong correlation between the variables, perhaps the most important thing to look for is to find an equation that shows this relationship mathematically. Different models have been used in research, but due to the high predictability of fractal-based models, these models are superior to other mathematical models [30]. In order to obtain a simple linear model with the least number of variables, regression between the fractal dimension of Higuchi sound signals and brain signals was performed separately in each band. According to the results, the two alpha and beta bands had the greatest effect in this regard. The influence of alpha and beta bands on external stimuli has also been expressed in other studies [43, 39]. Changes in alpha and beta bands are considered as a criterion for diagnosing drowsiness, fatigue, stress, etc. [45, 44].

Although according to Equation $8,98.3 \%$ of the sound changes can be explained by the two variables alpha and beta, a more accurate answer was obtained using the three variables alpha, beta and theta, which had the highest correlation. Using the obtained models, it is possible to study the characteristics of brain signals in response to sound changes. Because the recording of brain signals must be done under certain conditions and will usually be time consuming, we will be able to predict the characteristics of brain signals without recording them by finding the equations governing the relationships of external stimuli and the reaction of individuals during work.

\section{Conclusion}

This study showed that people's reactions, when exposed to sound, can be predicted using the fractal dimension. Therefore, it is possible to estimate the characteristics of brain signals without recording them, which are often costly and timeconsuming.

\section{Acknowledgement}

This study was the result of a research project numbered 15001/97, for which we thank the Vice Chancellor for Research and Technology of Arak University. Arak University of Technology is also appreciated for providing some of the test equipment.

\section{Conflict of Interest}

The authors declared no conflict of interest. 


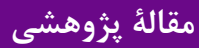

\section{همبستگى سيگَنال هاى مغزى و سيحَنال هاى صداى تراكتور مبتنى بر تحليل فركتال

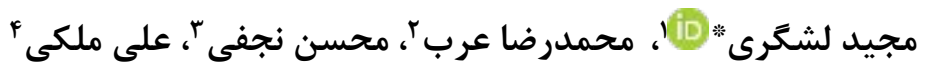

ا . دانشيار، گروه مهندسى مكانيك بيوسيستم، دانشكده كشاورزى و محيط زيست، دانشعاه اراك، اراك، ايران

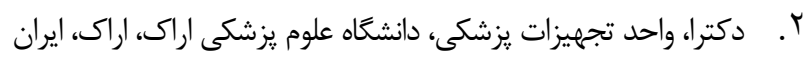

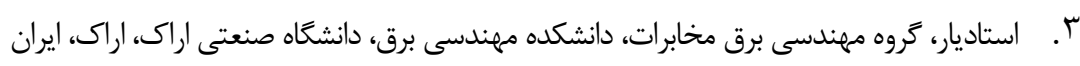

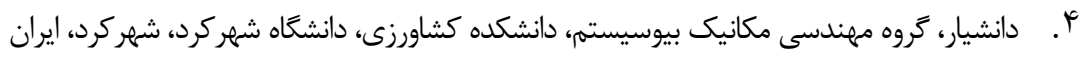

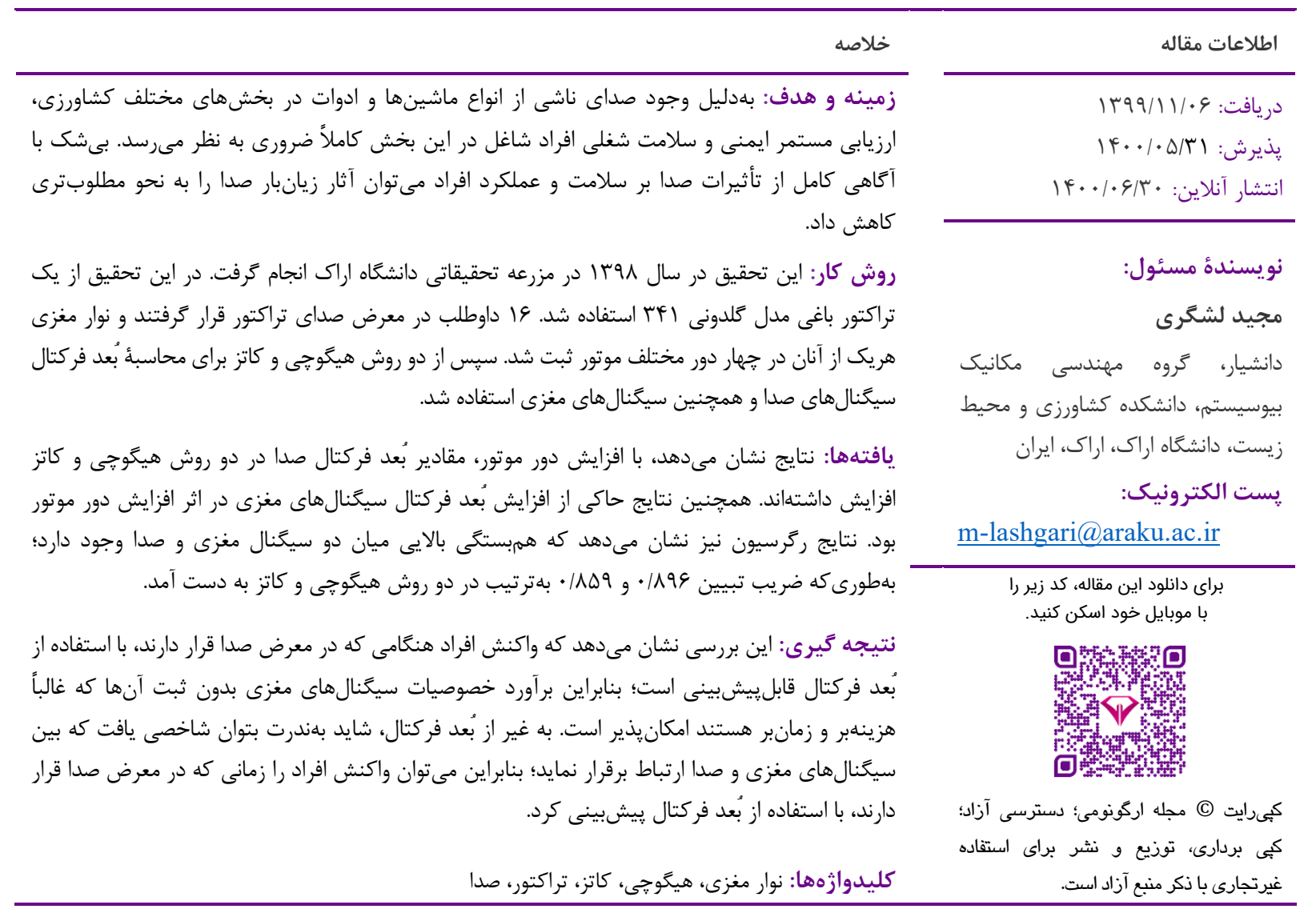

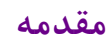

بيمارى قلبى- عروقى اشاره كرد [F-9]. اين يديده اثرات

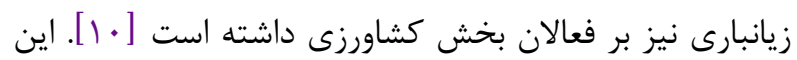

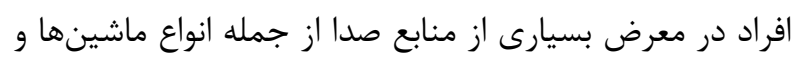

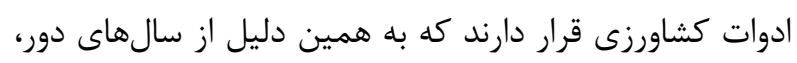
ابعاد مختلف آن توسط بسيارى از محققان بررسى شده و تا به ديه

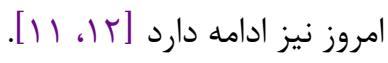

در دهdهاى اخير، موضوع مهمم ديخرى كه نظر محققان حوزء اركونومى را به خود جلب كرده است، ارزيابى تغييرات فيزيولوزيكى افراد در شرايط محيطى كار است [با إ]. بهمنظور
امروزه شايد ديخر نتوان محيط كار و زندگى بدون صدا را تصور كرد و يافتن جنين محيطى را بهويزه براى كار، رؤيا به

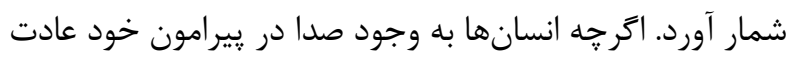
كردهاند، اثرات منفى آن بر سلامت و عملكرد آنان بر كسى بـ بـ

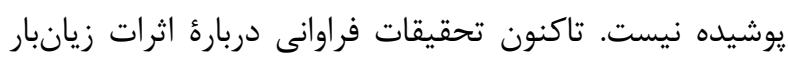
صدا بر سلامت جسم و روان انجام شده است [ـ-1 [1. از مهممترين اثرات نامطلوب صدا بر انسان علاوه بر افت شنوايى دائم و موقت

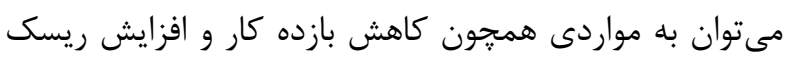
حوادث، اختلال در خواب، ايجاد ناراحتى هاى عصبى همونى - روانى و 
استفاده شده است. درواقع با استفاده از بُعد فركتال، بهترين

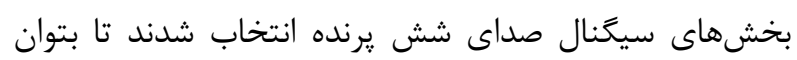

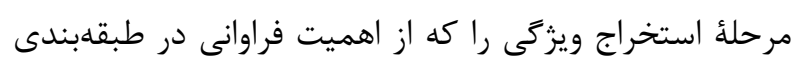

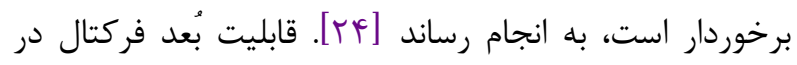

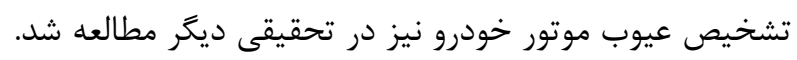

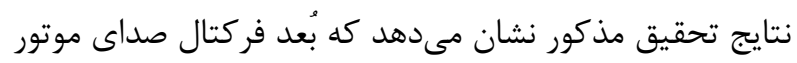

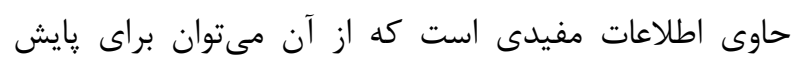

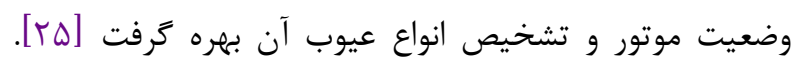

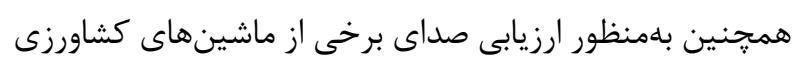

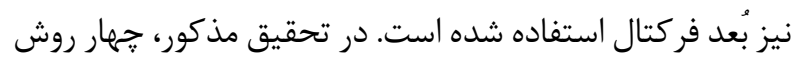

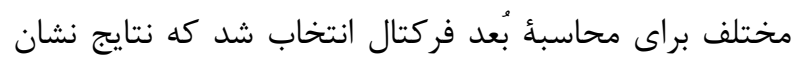

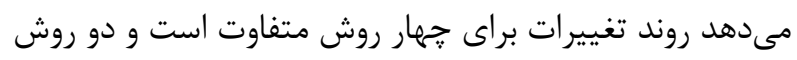

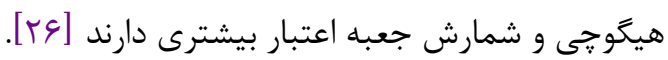

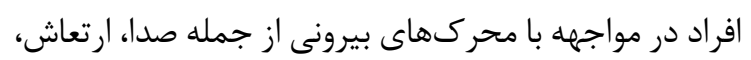
نور و غيره واكنشهايى نشان مىدهند كه با تحليل واكنشها

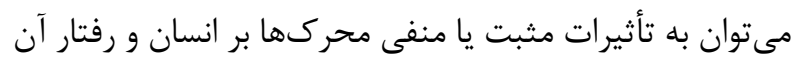

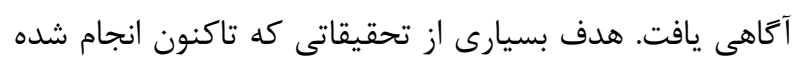

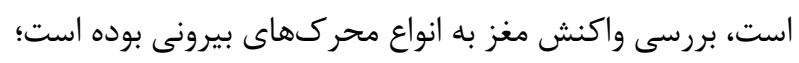

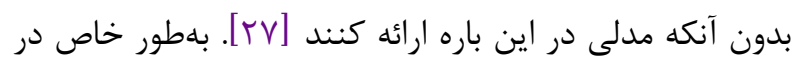
مقولهٔ محركهاى صوتى نيز مىتوان تحقيقاتى را يافت كه تندها

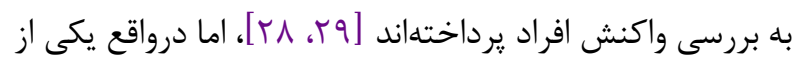
موضوعات مههم در اين مقوله، بررسى ارتباط خصوصيات

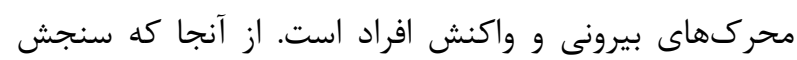

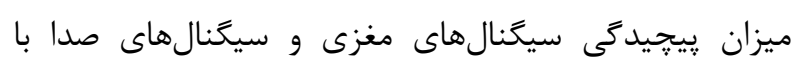

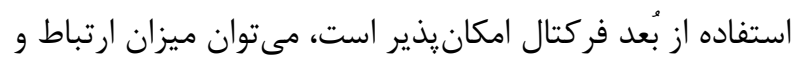

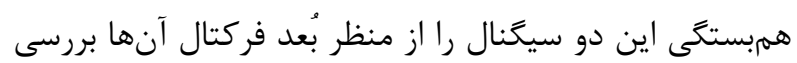

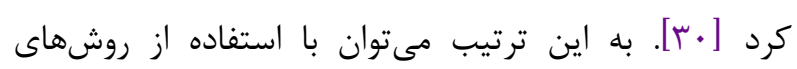

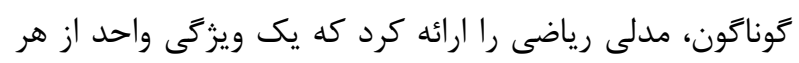

$$
\text { دو سيخنال را به هم مرتبط مى كنى رند. }
$$

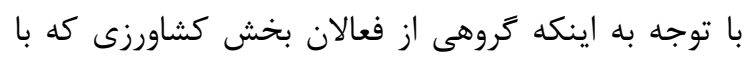

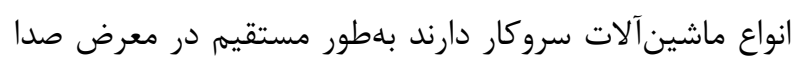

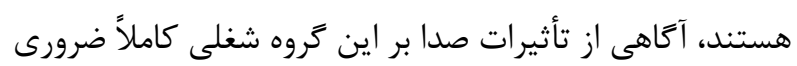

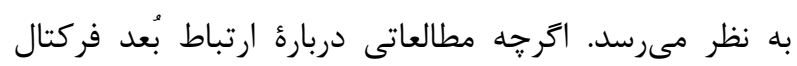

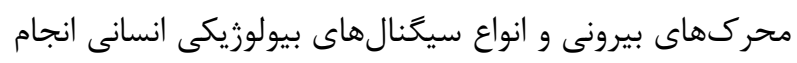

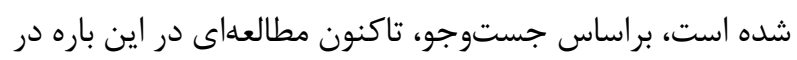

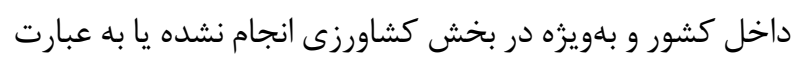
درستتر، نتايجى منتشر نشده است. با عنايت به اينكه از تراكتور

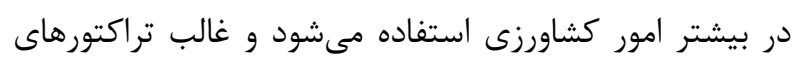

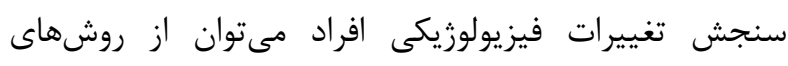

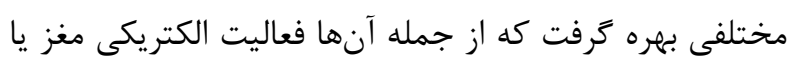

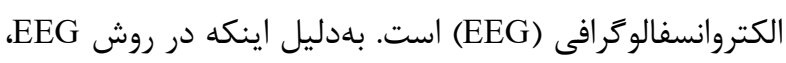

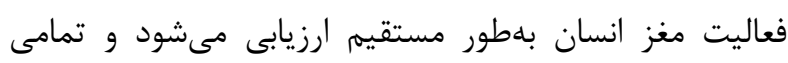

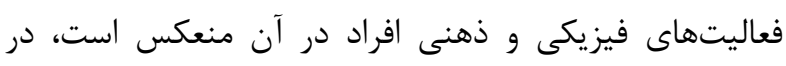

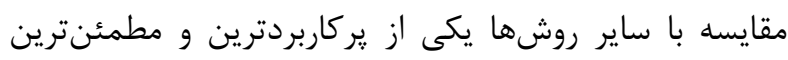

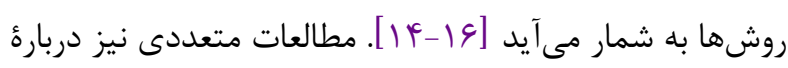
سنجش سيخنالهاى EEG در زمينههاى مختلف انجام شده

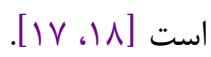

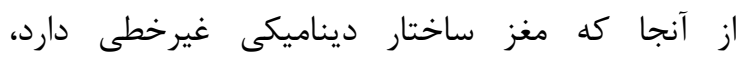

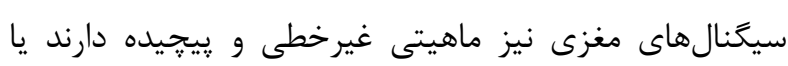

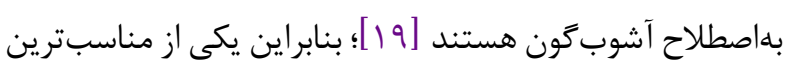

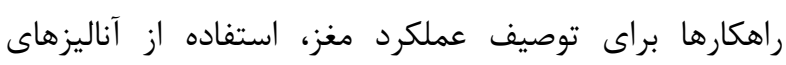

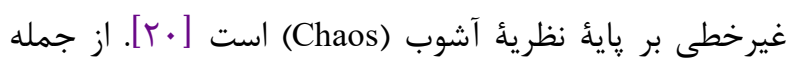

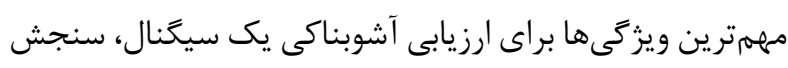

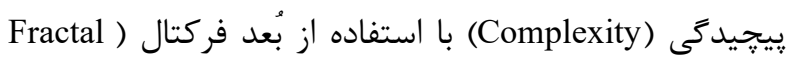
(Dimension

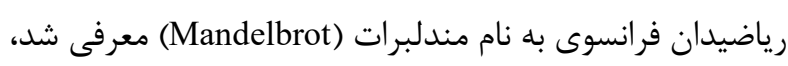

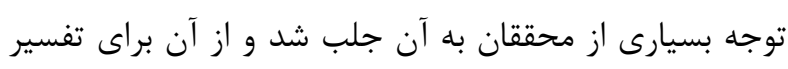

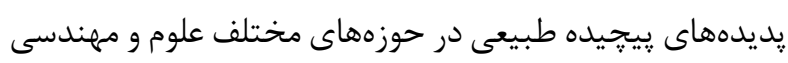

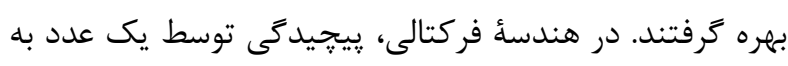

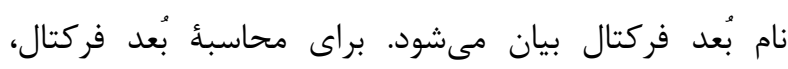

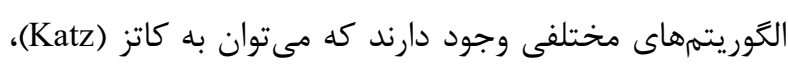

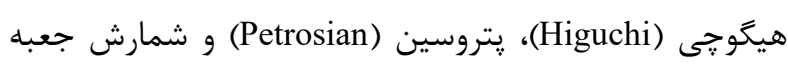
(Box Counting)

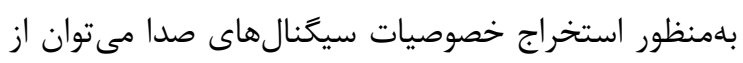

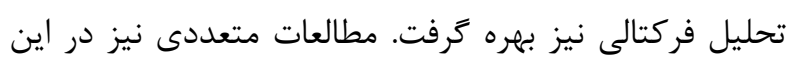

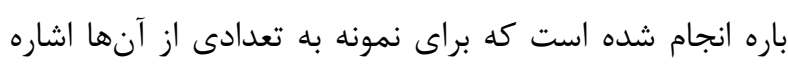

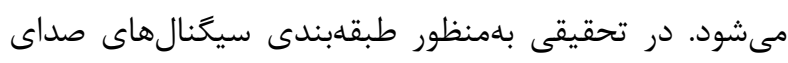

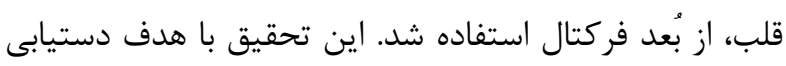

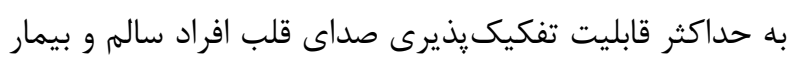

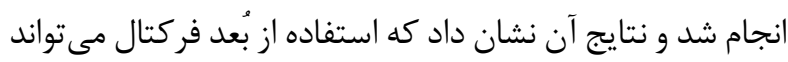

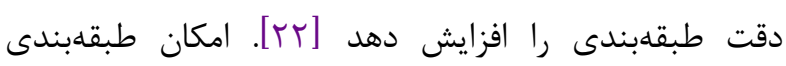

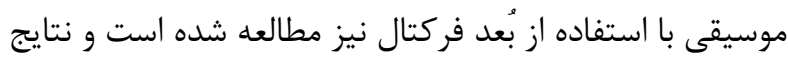

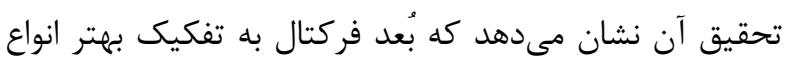

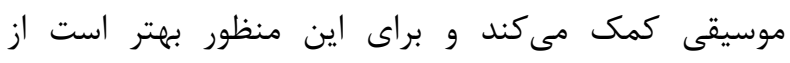

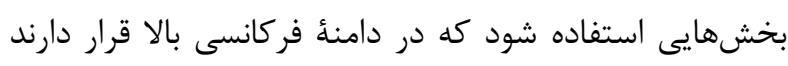

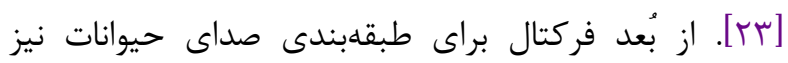


استاندارد • •r-· إخت شركت Science Beam استفاده شد. براى اين منظور، شانزده داوطلب با ميانخين سنى 9 ب سال كه همة آنان راستدست بودند، انتخاب شدند. معيار ورود شامل

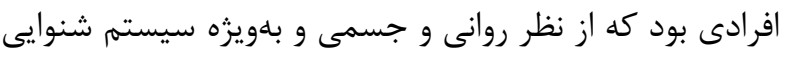

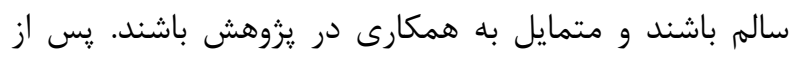

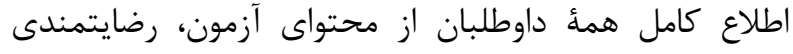

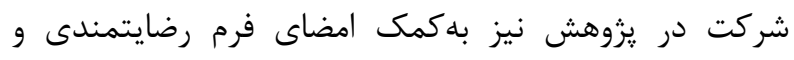

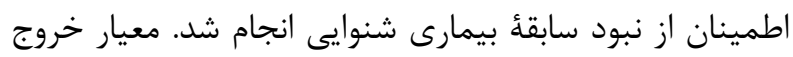

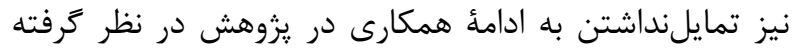

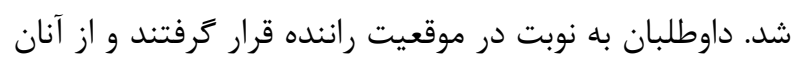

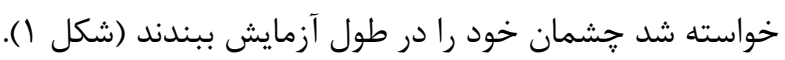

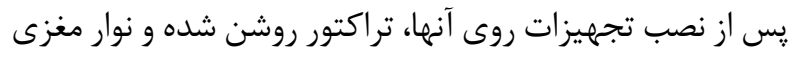

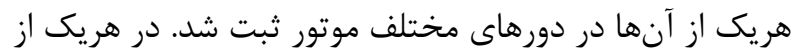

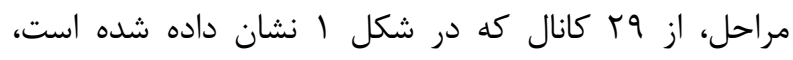

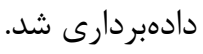
يس از ثبت دادهها، همهٔ آرتيفكتهاى مزاحم حذف شد. در

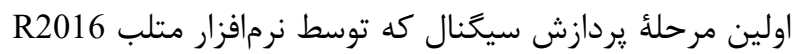

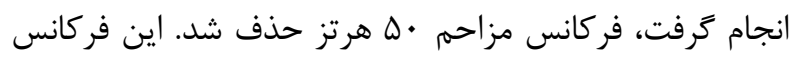
كه بهدليل وجود خطوط برق در نزديكى محل آزمايش ايجاد

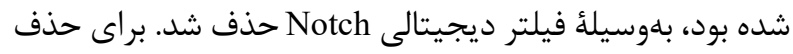

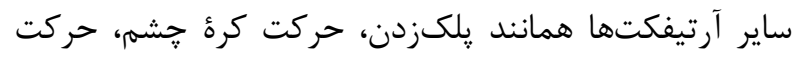
عضلات صورت، حركت الكترودها روى يوست سر كه كه عموماً

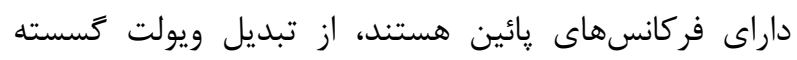

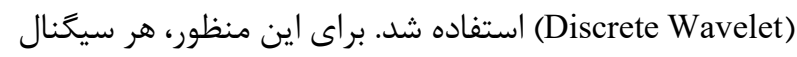
توسط تابع ويولت دابوجى db8 به هشت مرحله تجزيه شد.

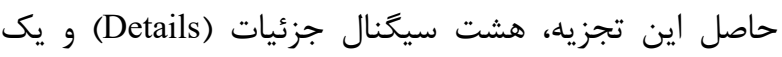

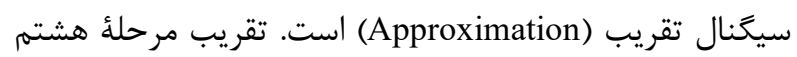

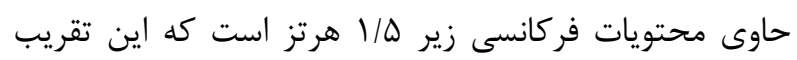

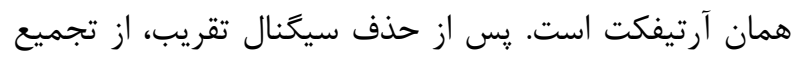

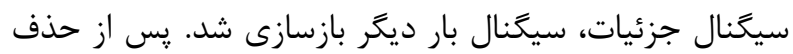

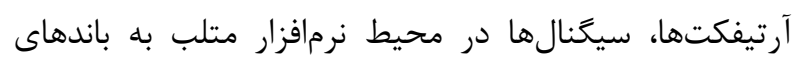

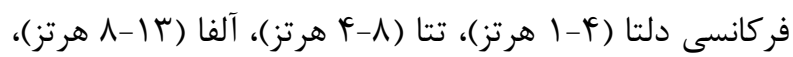

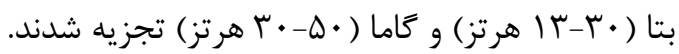

رايج كشور فاقد كابين هستند، هدف از اين تحقيق، بررسى

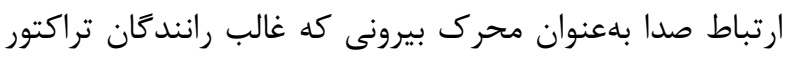

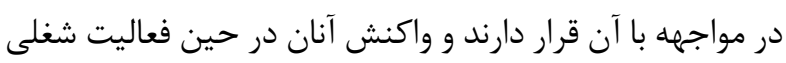
با استفاده از ارزيابى فعاليت مغزى است.

$$
\text { روش كار }
$$

\section{تجهيزات آزمايش}

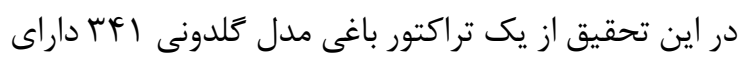

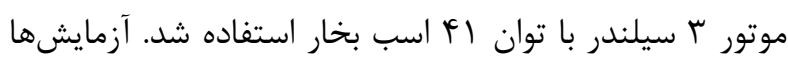

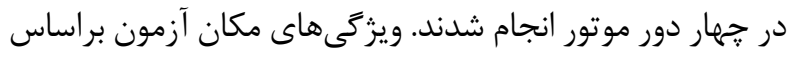

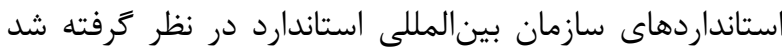

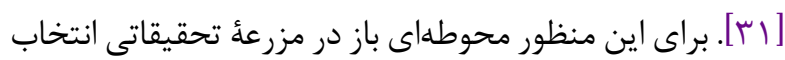

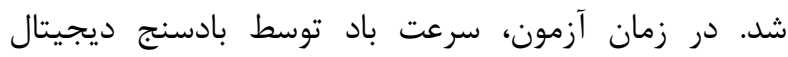

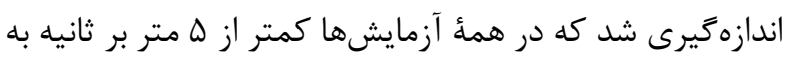

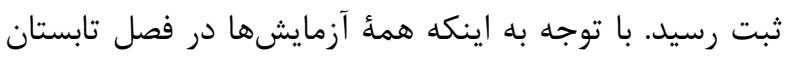

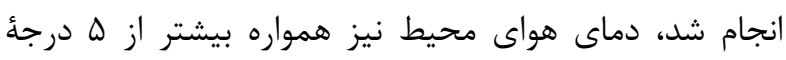

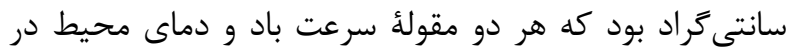

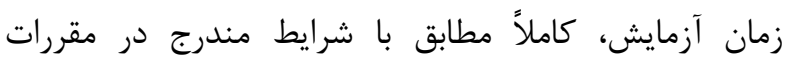

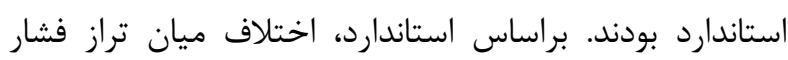

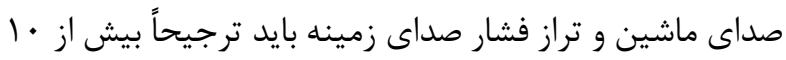

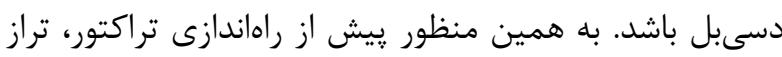

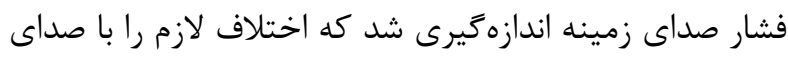
تراكتور داشت.

اندازهخيرى سيخَنال هاى صدا در موقعيت گَوش راننده انجام شد. براى اين منظور، ميكروفن روى سهِيايه در ارتفاعى متناسب

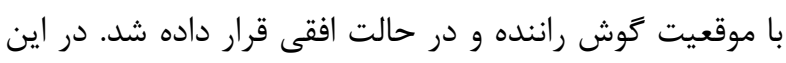

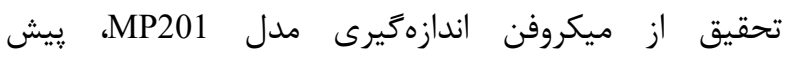

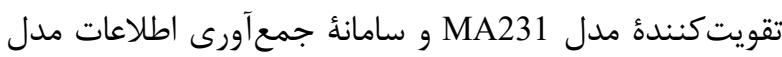

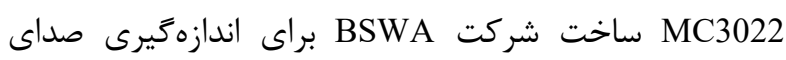
تراكتور استفاده شد. براى اندازهيرى سطح صدا، تركا، تراز معادل

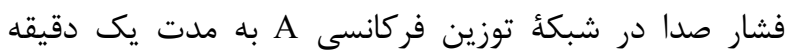
استفاده شد كه برحسب (AB(A) بيان مىشود. براى ثبت نوار مغزى از دستخاه ايران با نرخ نمونهبردارى ks/s 1 و رزولوشن بَ بر بيتى و كلاه 

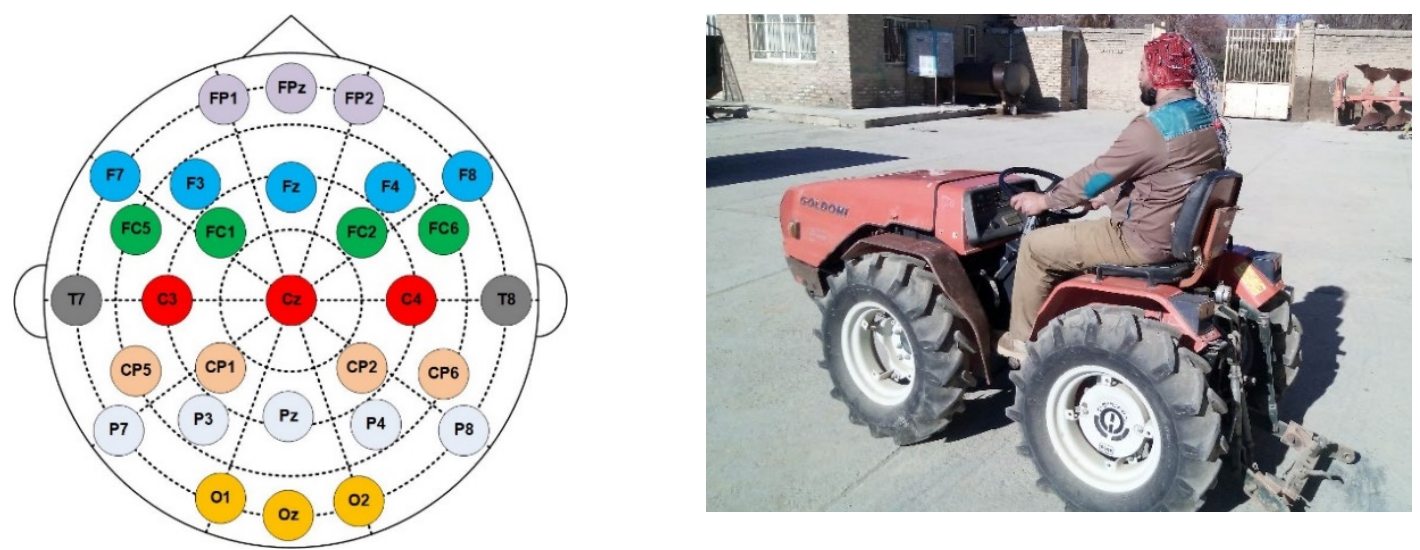

شكل ا. ثبت EEG با استفاده از كلاه استاندارد •؟r-1 (راست) و موقعيت الكترودها روى سر (جِّ)

در روش هيكوجى، بُعد فركتال طى مراحل زير محاسبه

$$
\text { مىشود [r [r]. }
$$

اگر (N) سرى زمانى تحت آناليز باشد، سرى

$$
\begin{aligned}
& \text { زمانى جديد } \\
& \text { رابطؤ }
\end{aligned}
$$

$X_{m}^{k}: x(m), x(m+k), x(m+2 k), \ldots, x\left(m+\right.$ int $\left.\left[\frac{(N-m)}{k}\right] k\right) \quad m=1,2, \ldots, k$

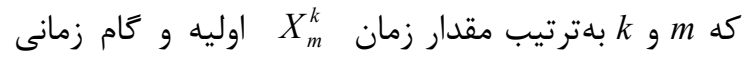
هستند. طول ميانكَين هر منحنى با استفاده از رابطة له محاسبه رابطه ه ه

$$
L_{m}(k)=\frac{1}{k}\left[\frac{N-1}{\operatorname{int}\left(\frac{N-m}{k}\right) k}\left(\sum_{i=1}^{m}\left(\frac{N-m)}{k}\right) x(m+i k)-x(m+(i-1) k)\right]\right.
$$

طول ميانگَين كل براى تمامى سرىهاى زمانى و براى همأ

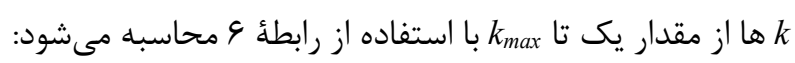

$$
L(k)=\frac{1}{k} \sum_{m=1}^{k} L_{m}(k) \quad \text { رابطو }
$$

در منحنى (ln)

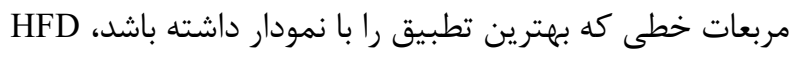

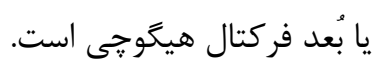

يافته ها

نتايج تراز فشار صداى تراكتور در دورهاى مختلف موتور در

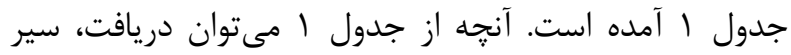

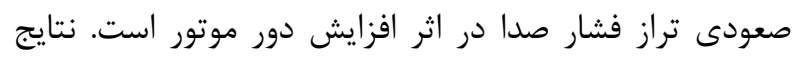

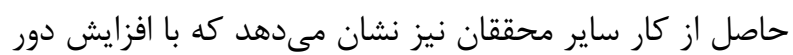

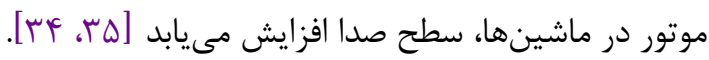

تُعد فر كتال

تاكنون روشهاى متعددى براى تعيين بُعد فركتال معرفى دئ

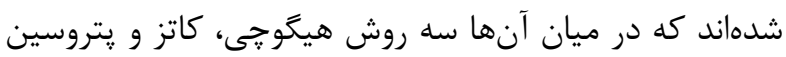
مقبوليت بيشترى داشتهاند و در بيشتر تحقيقات استفاده شدهاند

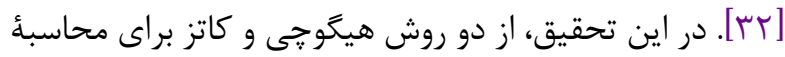

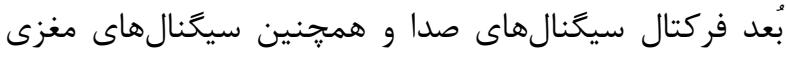

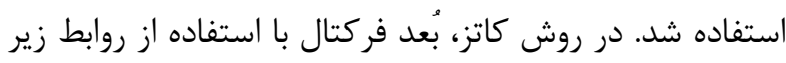

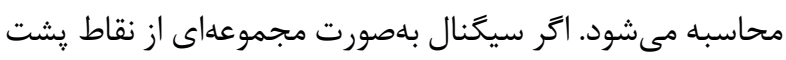

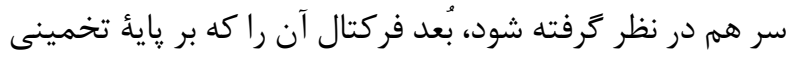

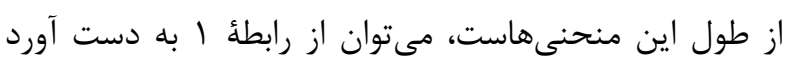
[بr]

$$
K F D=\frac{\log (L)}{\log (d)}
$$

رابطة |

كه L طول كلى منحنى و d قطر آن است. براى يك شكل

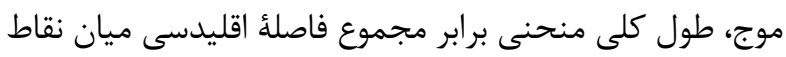

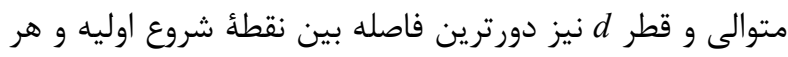

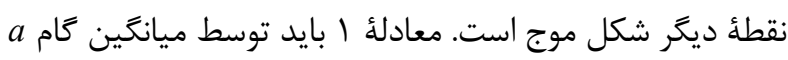

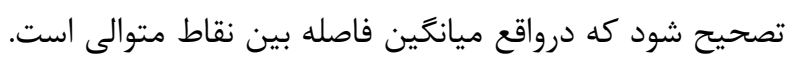

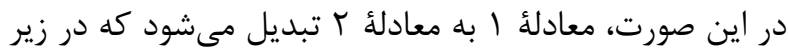

$$
K F D=\frac{\log (L / a)}{\log (d / a)}
$$

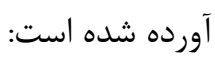

رابطة r

يس از تعريف n بهعنوان تعداد كامها در منحنى و قراردادن

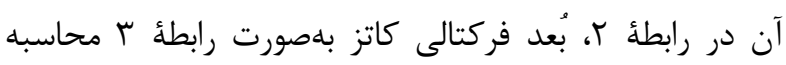

$$
\text { مىشود: - مى دان }
$$

$$
K F D=\frac{\log (n)}{\log (n)+\log (d / L)}
$$

رابطؤ r 
شدهاند. آنجه از اين شكل كاملاً مشخص است، با افزايش دور

موتور، مقادير بُعد فركتال در هر دو روش افز إيش دامد داشتهاند.
بُعد فركتال سيكنالهاى صداى تراكتور به دو روش كاتز و هيكوجى در دورهاى مختلف موتور در شكل r نشان داده

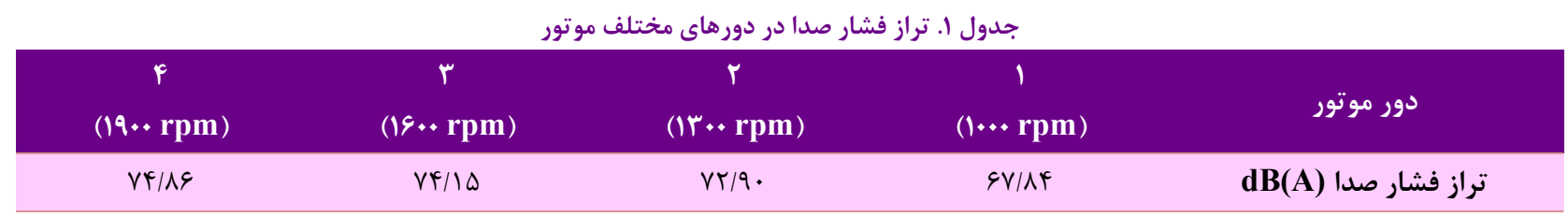
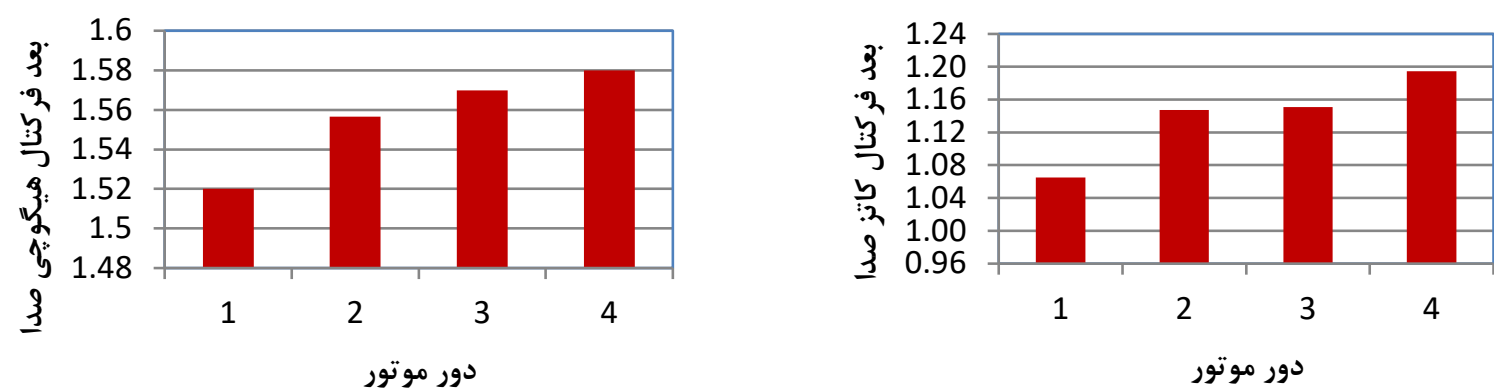

شكل r. تغييرات بُعد فركتال كاتز و هيكَوجى صداى تراكتور در دورهاى مختلف

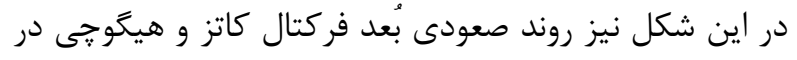

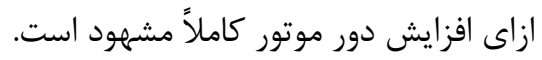

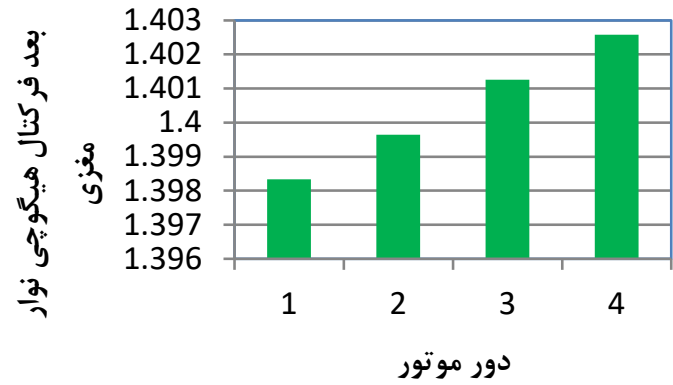

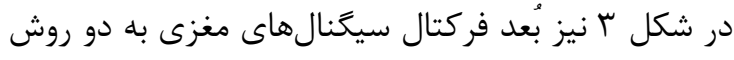
كاتز و هيكوجى در دورهاى مختلف موتور نشان داده شدهاند.

شكل r. تغييرات بُعد فركتال كاتز و هيكَوجى نوار مغزى در دورهاى مختلف موتور

r مى توان دريافت كه ضريب تبيين HFD از KFD بالاتر است.

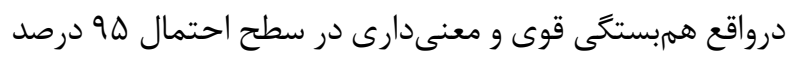
ميان HFD سيخنال هاى مغزى و صدا وجود دارد.

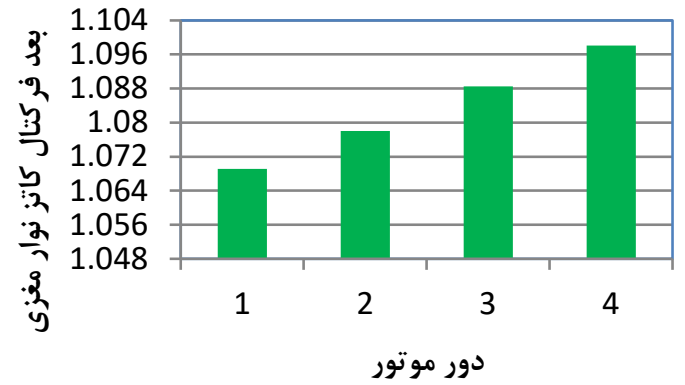

بامنظور بررسى ارتباط ميان صدا و فعاليت مغزى افراد مورد آزمون، رگرسيون ميان بُعد فركتال سيخَنال هاى مغزى و صدا

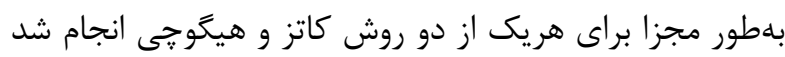

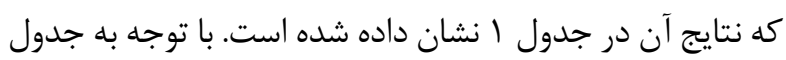

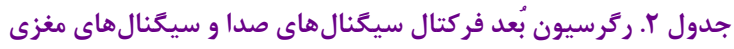

\begin{tabular}{|c|c|c|}
\hline HFD & KFD & \\
\hline - /199 & $\cdot / 1 \Delta \Delta 9$ & $\mathbf{R}^{2}$ \\
\hline IV/TGG & $\mid r / 1 \Lambda$ & $\mathbf{F}$ \\
\hline $.1 \cdot 4 q$ & $\cdot / \cdot V r$ & p-value \\
\hline
\end{tabular}


تغييرات بُعد فركتال هيكوجى هريك از باندهاى فر كانسى دلتا،

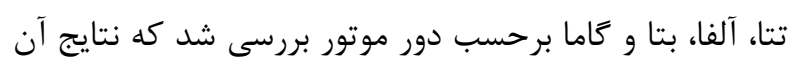
در شكل ع ارائه شده است.
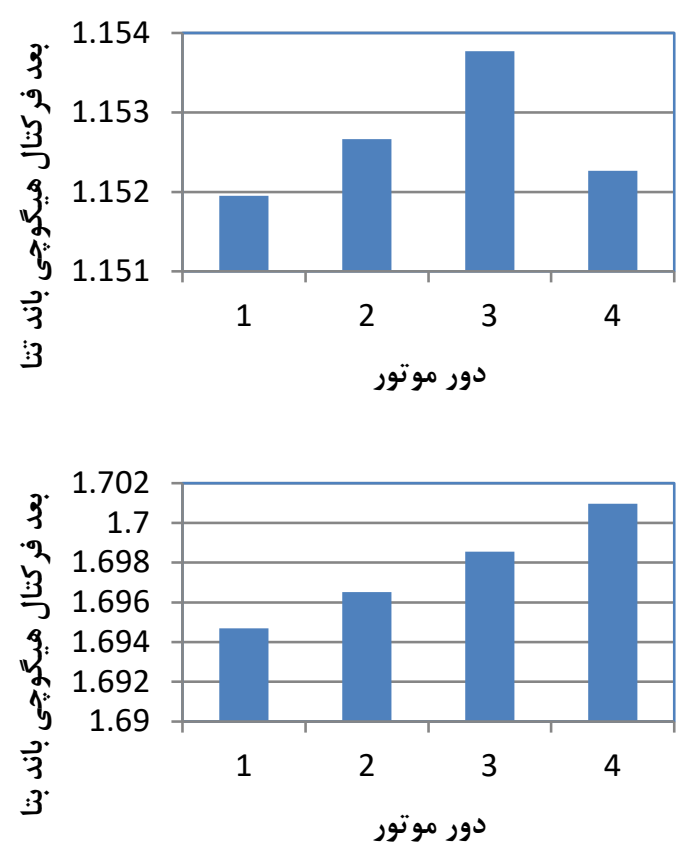

با توجه به اينكه همبستخى قوى و معنى دارى ميان HFD سيگنالهاى مغزى و صدا مشاهده شد، ميزان همببستكى در باندهاى فركانسى نيز بررسى شد. براى اين منظور ابتدا روند
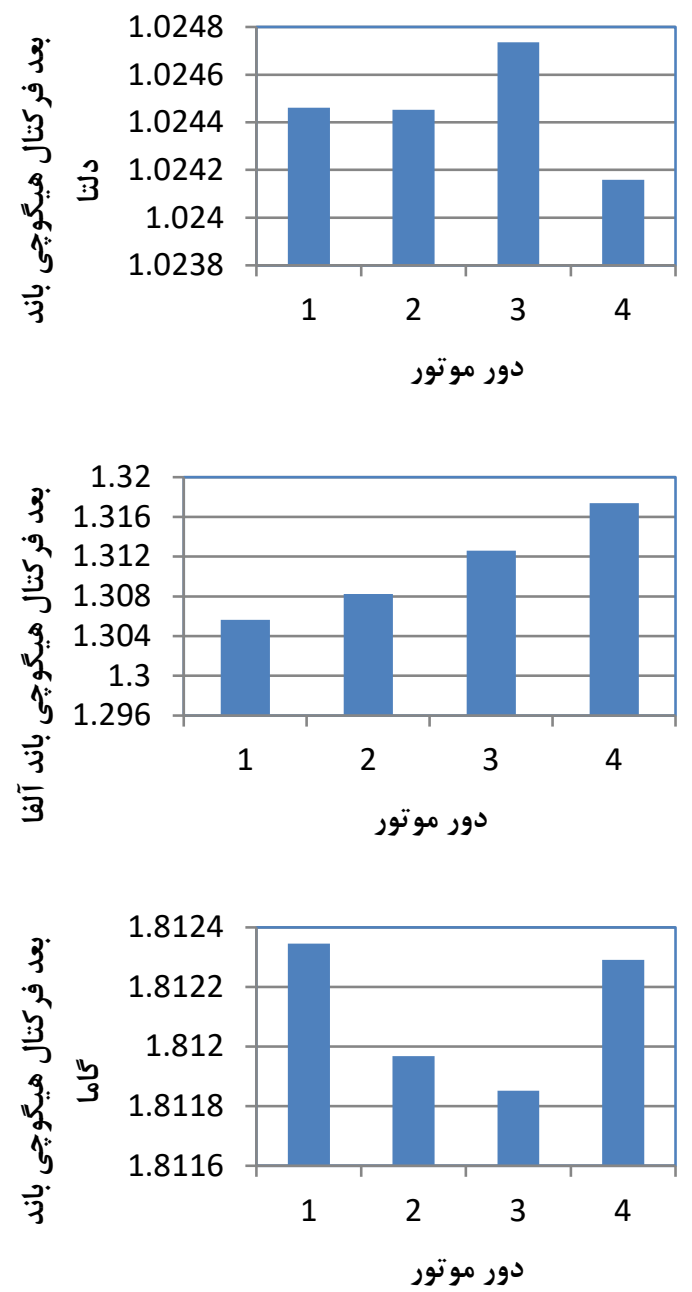

شكل f. روند تغييرات بُعد فركتال هيعَوجى سيكَنال هاى مغزى براى باندهاى فركانسى

با توجه به اين نتايج مىتوان دريافت كه همبستگى قوى بين

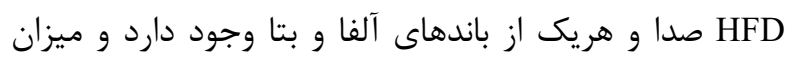
همبستخى در ساير باندها نيز ضعيف است.
از آنجا كه بُعد فركتال هيكوجى هريك از باندهاى فر كانسى

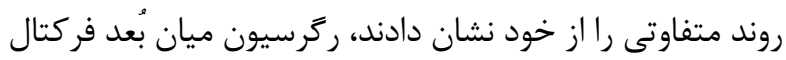
هيگوجى سيخَنالهاى صدا و سيخنالهاى مغزى در هريك از باندها به تفكيك انجام شد كه نتايج آن در جدول بآ آمده است.

\begin{tabular}{|c|c|c|c|c|c|}
\hline$\gamma$ & $\beta$ & $\alpha$ & $\boldsymbol{\theta}$ & $\delta$ & \\
\hline.$/ 191$ & $\cdot|A \vee|$ & $\cdot|A T|$ & - ITAT & $\cdot 1 \cdot r \Delta$ & $\mathbf{R}^{2}$ \\
\hline$\cdot / f \cdot r$ & IT/FAF & $q / / \vee \wedge$ & $.19 \vee 9$ &.$/ \cdot V r$ & $\mathbf{F}$ \\
\hline .109. & $.1 .9 \mathrm{~V}$ & .1 .94 &.$/ 4 q V$ & . $|\mathrm{A}| \mathrm{T}$ & p-value \\
\hline
\end{tabular}


تبيين هريك از مدلها بيانكر اين است كه متغيرهاى مستقل،

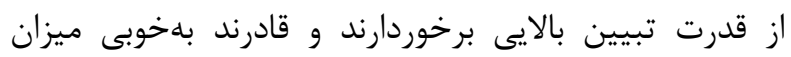
تغييرات متغير وابسته را تبيين كنند. تئن برخودرند

Sound $=+4.587 \alpha-4.457$

Sound $=-23.061 \alpha+53.140 \beta-58.425$

Sound $=-19.188 \alpha+45.330 \beta+4.913 \theta-55.908$

مقايسه با ساير روشها از جمله كاتز نيز در ساير تحقيقات تأييد شده

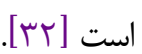

وجود همبستخى ميان بُعد فركتال محركهاى بيرونى و واكنش آنش

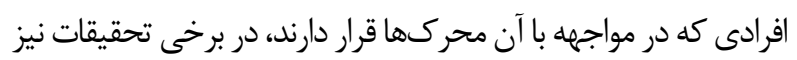

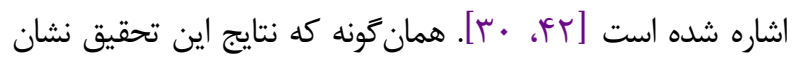

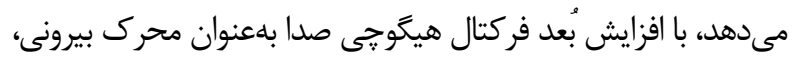

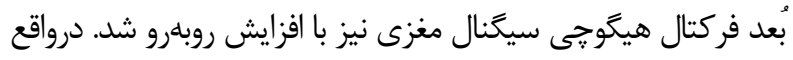

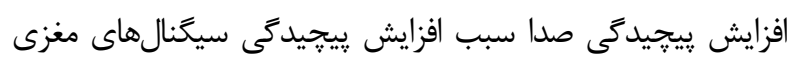

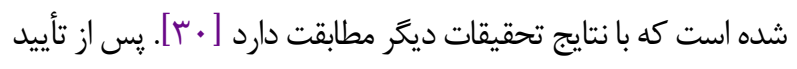

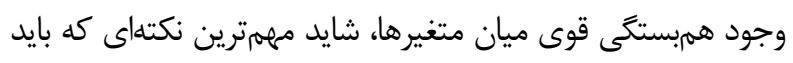

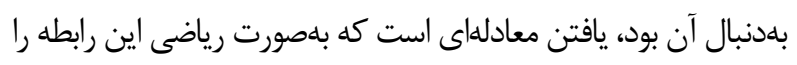

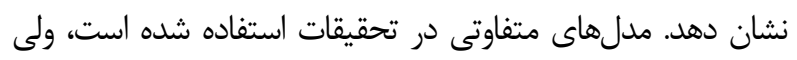

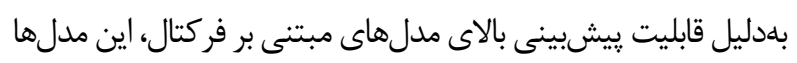

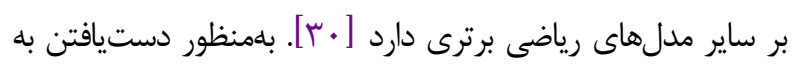

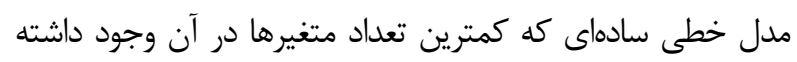

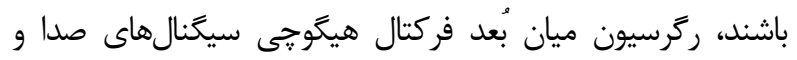

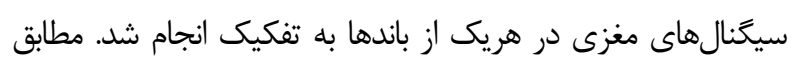

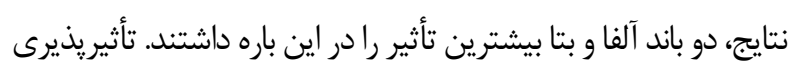

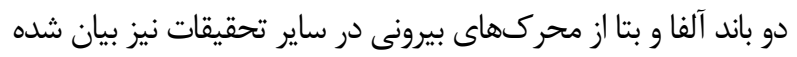

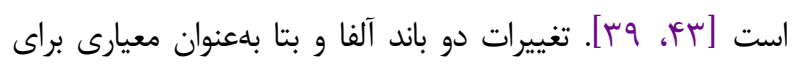
تشخيص خوابآلودىى، خستخى، استرس و غيره در نظر كرفته

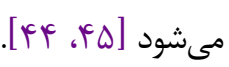

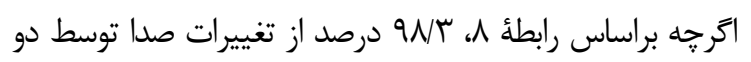

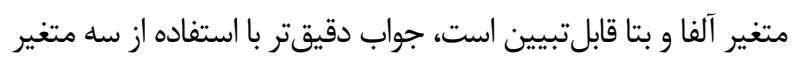
آلفا، بتاو تتا حاصل شده است كه بيشترين ميزان همبستخى رأ را داشتند.

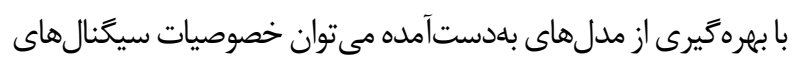

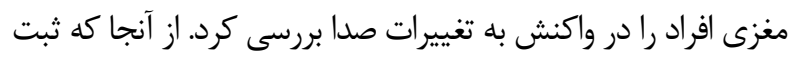
سيكنال هاى مغزى بايد تحت شرايط خاصى انجام شود و معمولاً نيز

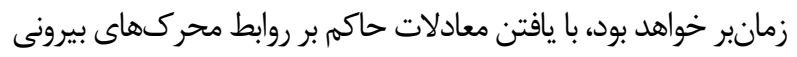

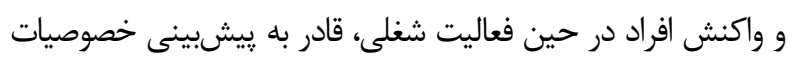
سيكنال هاى مغزى بدون ثبت آنها خواهيم بود.
با استفاده از ركرسيون جندمتغيره، سه مدل خطى در قالب

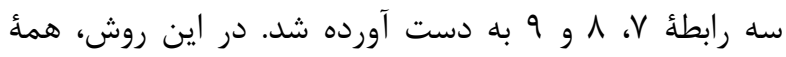

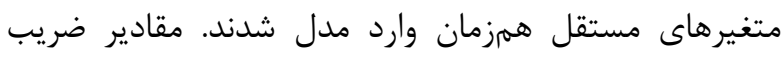
• • رابطة • رابطة $990 \Delta R^{2}=$

براساس رابطة V، 1/ N درصد از تغييرات صدا را تنها متغير آلفا

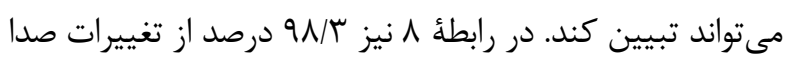

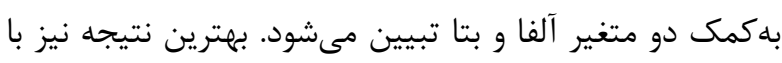

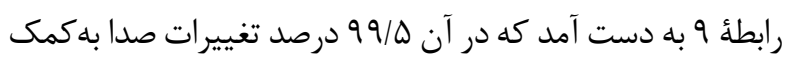

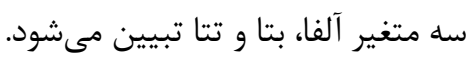

بحث

نتايج اين تحقيق حاكى از آن است كهُ بُعد فركتال سيكنالهاى

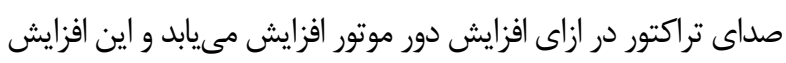

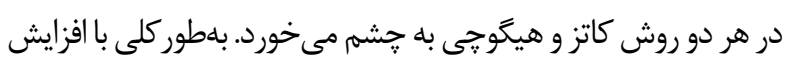

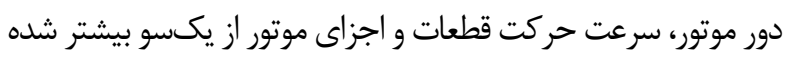

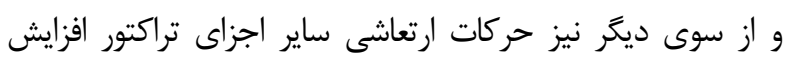

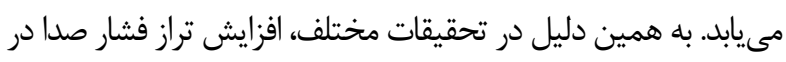

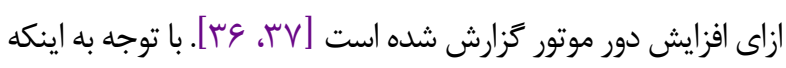

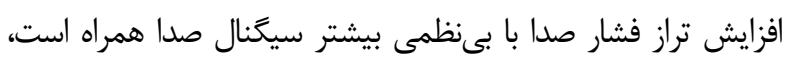

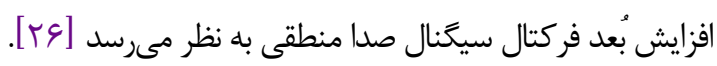

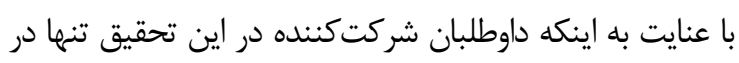

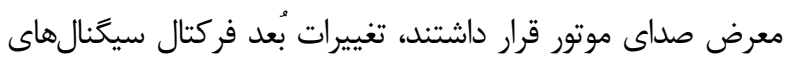

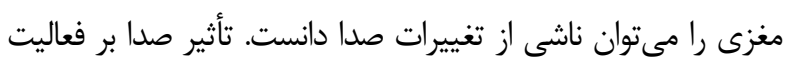

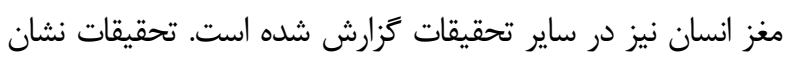

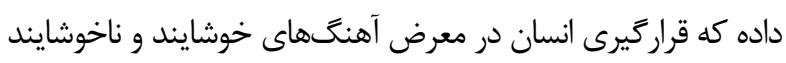

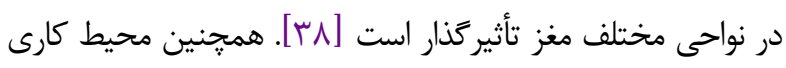

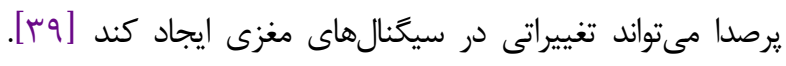

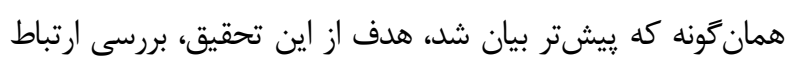

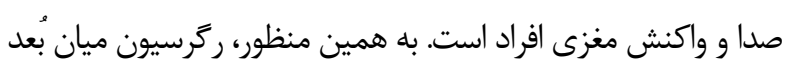

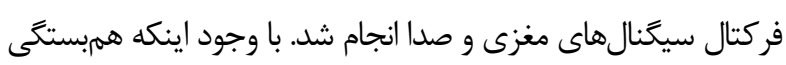

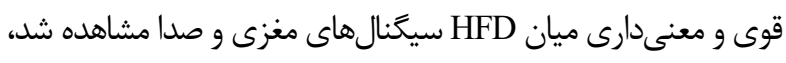

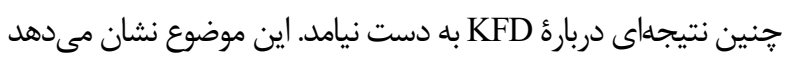

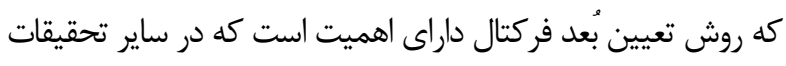

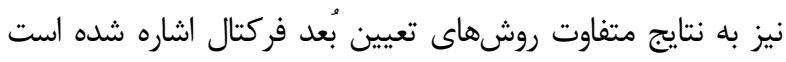

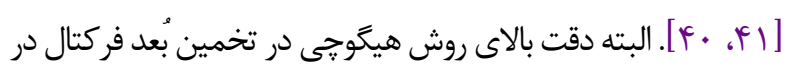




$$
\begin{aligned}
& \text { كيرد كه تراكتور در حال حركت باشد كه نيازمند تجهيزات يرتابل }
\end{aligned}
$$

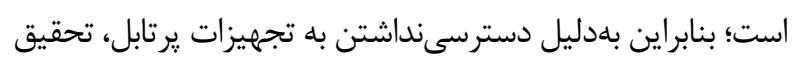

$$
\begin{aligned}
& \text { حاضر با محدوديت همراه بوده است. } \\
& \text { تقدير و تشكر }
\end{aligned}
$$

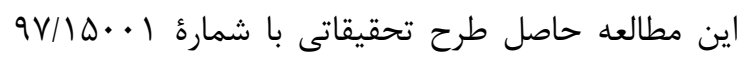

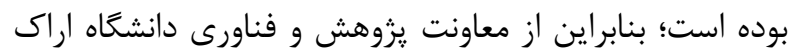

$$
\begin{aligned}
& \text { سياسگزارى مىشود. همجنين از دانشگاه صنعتى اراك بهدليل } \\
& \text { تأمين بخشى از تجهيزات آزمايش تقدير و تشكر مىشودي } \\
& \text { تعارض منافع } \\
& \text { ميان نويسندكان هيجزَّهن تعارضى در منافع وجود ندارد. } \\
& \text { سمهم نويسند } \\
& \text { تمام نويسندكان در طراحى، اجرا و تحليل اين مطالعه } \\
& \text { مشاركت داشتهاند. } \\
& \text { منابع مالى } \\
& \text { منابع مالى اين مطالعه را دانشعاه اراك تأمين كرده است. } \\
& \text { نتيجه گَيرى }
\end{aligned}
$$

\section{References}

1. Basner M, Babisch W, Davis A, Brink M, Clark C, Janssen S, Stansfeld S. Auditory and non-auditory effects of noise on health. The lancet. 2014;383(9925):1325-32. [DOI:10.1016/S0140-6736(13)61613-X]

2. Sygna K, Aasvang GM, Aamodt G, Oftedal B, Krog NH. Road traffic noise, sleep and mental health. Environ Res. 2014;131:17-24. [DOI:10.1016/j.envres.2014.02.010] [PMID]

3. Van Kamp I, Davies H. Noise and health in vulnerable groups: a review. Noise and health. 2013;15(64):153. [DOI:10.4103/1463-1741.112361] [PMID]

4. Gorai AK, Pal AK. Noise and its effect on human beingA review. J Environ Sci Eng. 2006;48(4):253.

5. Nassiri P, Monazam M, Dehaghi BF, Abadi LI, Zakerian SA, Azam K. The effect of noise on human performance: A clinical trial. Int J Occup Environ Med (The IJOEM). 2013;4:212-87.

6. Van Kempen EE, Kruize H, Boshuizen HC, Ameling CB, Staatsen BA, de Hollander AE. The association between noise exposure and blood pressure and ischemic heart disease: a meta-analysis. Environ Health Perspect. 2002;110(3):307-17. [DOI:10.1289/ehp.02110307] [PMID] [PMCID]
7. Stansfeld SA, Matheson MP. Noise pollution: nonauditory effects on health. Br Med Bull. 2003;68(1):24357. [DOI:10.1093/bmb/ldg033] [PMID]

8. Clark C, Stansfeld SA. The effect of transportation noise on health and cognitive development: A review of recent evidence. Int J Comp Psychol. 2007;31;20(2).

9. Seidman MD, Standring RT. Noise and quality of life. Int J Environ Res Public Health. 2010;7(10):3730-8. [DOI:10.3390/ijerph7103730] [MID] [PMCID]

10. Lar MB, Pay M, Bagheri J, Pour ZK. Comparison of noise level of tractors with cab and without in different gears on driver ear and bystander. Afr J Agric Res. 2012;7(7):1150-5. [DOI:10.5897/AJAR11.1431]

11. Ghaderi M, Javadikia H, Naderloo L, Mostafaei M, Rabbani H. Analysis of noise pollution emitted by stationary MF285 tractor using different mixtures of biodiesel, bioethanol, and diesel through artificial intelligence. Environ Sci Pollut Res. 2019;26(21):2168292. [DOI:10.1007/s11356-019-05523-1] [PMID]

12. Lalremruata, Dewangan KN, Patel T. Noise exposure to tractor drivers in field operations. Int $\mathrm{J}$ Veh Perform. 2019;5(4):430-42. [DOI:10.1504/IJVP.2019.104085] 
13. Lashgari M, Arab MR. Investigation of relationship between noise annoyance and neurophysiological responses of drivers in exposure to tractor sound. Iran J Ergon. 2018;6(3):65-74. [DOI:10.30699/jergon.6.3.7]

14. Liu J, Zhang C, Zheng C. EEG-based estimation of mental fatigue by using KPCA-HMM and complexity parameters. Biomedical Signal Processing and Control. 2010;5(2):124-30. [DOI:10.1016/j.bspc.2010.01.001]

15. Li W, He QC, Fan XM, Fei ZM. Evaluation of driver fatigue on two channels of EEG data. Neurosci Lett. 2012;506(2):235-9. [DOI:10.1016/j.neulet.2011.11.014] [PMID]

16. Chen C, Li K, Wu Q, Wang H, Qian Z, Sudlow G. EEGbased detection and evaluation of fatigue caused by watching 3DTV. Displays. 2013;34(2):81-8. [DOI:10.1016/j.displa.2013.01.002]

17. Rasmussen P, Stie H, Nybo L, Nielsen B. Heat induced fatigue and changes of the EEG is not related to reduced perfusion of the brain during prolonged exercise in humans. J Therm Biol. 2004 Oct 1;29(7-8):731-7. [DOI:10.1016/j.jtherbio.2004.08.047]

18. Bachmann M, Lass J, Hinrikus H. Single channel EEG analysis for detection of depression. Biomed Signal Process Control. $2017 ; 31: 391-7$. [DOI:10.1016/i.bspc.2016.09.010]

19. Rodriguez-Bermudez G, Garcia-Laencina PJ. Analysis of EEG signals using nonlinear dynamics and chaos: a review. Appl Math Inf Sci. 2015;9(5):2309.

20. Khodabakhshi M, Saba V. The Analysis of Individuals Emotions Through Brain Signals Using Poincare Approach. Paramed Sci Mil Health. 2018;13(3):12-9.

21. Mohammadi E, Kermani S, Golparvar M. Evaluation of Chaos on Electroencephalogram in Different Depths of Anesthesia. J Isfahan Med Sch. 2018; 36(482): 601-6.

22. Hamidi M, Ghassemian H, Imani M. Classification of heart sound signal using curve fitting and fractal dimension. Biomedical Signal Processing and Control. 2018;39:351-9. [DOI:10.1016/j.bspc.2017.08.002]

23. Bigerelle M, Iost A. Fractal dimension and classification of music. Chaos, Solitons \& Fractals. 2000;11(14):217992. [DOI:10.1016/S0960-0779(99)00137-X]

24. Gunasekaran S, Revathy K. Automatic recognition and retrieval of wild animal vocalizations. Int $\mathrm{J}$ Comput Theory Eng. 2011 Feb 1;3(1):136. [DOI:10.7763/IJCTE.2011.V3.295]

25. Chen J, Liao S, Gan J, Wang XW, Zhu LJ, Mi L. Application of Fractal Dimension to Engine Fault Diagnosis Based on Noise. Vehicle Engine. 2011; (5):21.

26. Boroujeni FM, Maleki A. Fractal Analysis of Noise Signals of Sampo and John Deere Combine Harvesters in Operational Conditions. Archives of Acoustics. 2019;44. [DOI:10.2307/j.ctvk8w13s.43]

27. Namazi H, Kulish VV. Fractional diffusion based modelling and prediction of human brain response to external stimuli. Comput Math Methods Med. 2015; 2015:1-11. [DOI:10.1155/2015/148534] [PMID] [PMCID]
28. Will U, Berg E. Brain wave synchronization and entrainment to periodic acoustic stimuli. Neurosci Lett. 2007;424(1):55-60. [DOI:10.1016/j.neulet.2007.07.036] [PMID]

29. Eswaran H, Draganova R, Preissl H. Auditory evoked responses: a tool to assess the fetal neurological activity. Appl Acoust. 2007;68(3):270-80 [DOI:10.1016/j.apacoust.2006.03.004]

30. Namazi H. Complexity based analysis of the correlation between external stimuli and bio signals. ARC J. Neurosci.. 2018;3(3):6-9.

31. ISO 5131. 1996. Acoustics: Tractors and machinery for agriculture and forestry measurement of noise at operator's position.

32. Kesić S, Spasić SZ. Application of Higuchi's fractal dimension from basic to clinical neurophysiology: a review. Computer methods and programs in biomedicine. 2016 Sep 1;133:55-70. [DOI:10.1016/i.cmpb.2016.05.014] [PMID]

33. Abdossalehi, M., Nasrabadi, A. M. \& Firouzabadi, S.M. Investigation of Positive, Negative and Neutral Emotion's determinism through EEG signal processing in extracted component of ICA. Iran J Biomed Eng. 2013;7(2):143153.

34. Rabbani H, Lorestani A, Javadikia P, Gholami R. Noise evaluation of MF285 tractor while pulling a trailer in an asphalt road. Int J Agric Eng. 2012;14(4):50-5.

35. Ghotbi MR, Monazzam MR, Khanjani N, Nadri F, Fard SM. Driver exposure and environmental noise emission of Massey Ferguson 285 tractor during operations with different engine speeds and gears. Afr J Agric Res. 2013;8(8):652-659.

36. Jahanbakhshi A, Ghamari B, Heidarbeigi K. Effect of engine rotation speed and gear ratio on the acoustic emission of John Deere 1055I combine harvester. Int J Agric Eng. 2016;18(3):106-12.

37. Lashgari M, Maleki A. Evaluation of lawn tractor noise using acoustic and psychoacoustic descriptors. Eng Agric Environ Food. 2016;9(1):116-22. [DOI:10.1016/i.eaef.2015.07.001]

38. Banerjee A, Sanyal S, Patranabis A, Banerjee K, Guhathakurta T, Sengupta R, Ghosh D, Ghose P. Study on brain dynamics by non linear analysis of music induced EEG signals. Phys A: Stat Mech Appl. 2016 Feb 15;444:110-20. [DOI:10.1016/j.physa.2015.10.030]

39. Bhoria R, Singal P, Verma D. Analysis of effect of sound levels on EEG. Int $\mathrm{J}$ Adv Technol Eng. 2012 Mar;2(2):121-4.

40. Pavithra M, NiranjanaKrupa B, Sasidharan A, Kutty BM, Lakkannavar M. Fractal dimension for drowsiness detection in brainwaves. In 2014 International Conference on Contemporary Computing and Informatics (IC3I) 2014 Nov 27 (pp. 757-761). IEEE. [DOI:10.1109/IC3I.2014.7019676]

41. Ahmadlou M, Adeli H, Adeli A. Fractality analysis of frontal brain in major depressive disorder. Int $\mathrm{J}$ Psychophysiol. 2012 Aug 1;85(2):206-11. [DOI:10.1016/j.ijpsycho.2012.05.001] [PMID] 
42. Alipour ZM, Khosrowabadi R, Namazi H. Fractal-based analysis of the influence of variations of rhythmic patterns of music on human brain response. Fractals. 2018;26(05):1850080.

[DOI:10.1142/S0218348X18500809]

43. Yeo MV, Li X, Shen K, Wilder-Smith EP. Can SVM be used for automatic EEG detection of drowsiness during car driving? Safety Science. 2009;47(1):115-24. [DOI:10.1016/j.ssci.2008.01.007]
44. Jap BT, Lal S, Fischer P, Bekiaris E. Using EEG spectral components to assess algorithms for detecting fatigue. Expert Syst. Appl. 2009;36(2):2352-9. [DOI:10.1016/j.eswa.2007.12.043]

45. Sulaiman N, Taib MN, Lias S, Murat ZH, Aris SA, Hamid $\mathrm{NH}$. Novel methods for stress features identification using EEG signals. International Journal of Simulation: Systems, Science and Technology. 2011;12(1):27-33. 